\title{
Resistance-Based Probabilistic Design by Order Statistics for an Oil and Gas Deep-water Well Casing String Affected by Wear During Kick Load
}

\author{
F. Di Maio, P. Baraldi, L. Brivio \\ Energy Department, Politecnico di Milano, Via la Masa 34, 20156 Milano, Italy
}

E. Zio

Energy Department, Politecnico di Milano, Via la Masa 34, 20156 Milano, Italy

Chair on Systems Science and the Energetic Challenge, Fondation EDF, Ecole Centrale Paris and Supelec, France

\author{
C. Magno \\ Petrobras, Brazil
}

\begin{abstract}
Deep-water wells for oil and gas extraction make structural components, such as casing and tubing, work in extremely harsh environmental conditions that accelerate component degradation and increase failure probability. Therefore, it is important to properly design casing strings under these operative circumstances. To do this, a Resistance-Based Probabilistic Design (RBPD) approach is here complemented with the quantification of the probabilistic safety margin that the design load does not exceed the casing strength. Wear is taken as the main degradation mechanism during drilling and the accidental event of a kick load is considered to affect the performance of the casing string. By so doing, we consider all uncertainties affecting the casing burst strength and evaluate the string safety by the Order Statistic (OS) method.
\end{abstract}

\section{INTRODUCTION}

The increase of oil demand and the consequent depletion of shallow conventional hydrocarbon reservoirs has led to the exploration and drilling of deep oil wells (Kumar et al. 2013) (Mitchell et al. 2012). Such High Pressure and High Temperature (HPHT) environment enhances the casing degradation mechanisms (e.g, wear, corrosion, yield strength deration) that may result in accidents with severe consequences (Kumar et al. 2013) (Baraldi et al. 2012a,b) (Abimbola et al. 2014). These consequences are to be avoided by design, ensuring casing integrity, during both perforation and production (Mitchell et al. 2012).

Casing design consists in the iterative process of (Blade Energy Partners 2005):

1) Identification of all possible load scenarios (e.g., kick, pressure tests, cementing, injection, changes in temperature, evacuation, buckling, etc.);

2) Calculation of loads $L$ (e.g., internal pressure, external pressure, axial force, torsion) at each depth of the casing string;
3) Calculation of casing strength $S$ under the current design;

4) Check that the strength is larger than loads: if not, modify the design and repeat step 3 ).

Different approaches to verify the well structural integrity and the exploitation of the reservoir in a safely and cost-effective manner are available (Blade Energy Partners 2005):

- Working Stress Design (WSD)

- Limit States Design (LSD)

- Resistance-Based Probabilistic Design (RBPD)

- Reliability-Based Design (RBD)

All these approaches aim at ensuring a reliable well design that minimizes the probability that the load exceeds the strength while minimizing the cost of the casing string. With respect to the abovementioned casing design iterative process, these approaches mainly differ in steps 2) 3) and 4), regarding the assumptions taken to calculate the strength and the load (listed in Table 1).

The safety of the design is done by checking if the design load $L$ is lower or larger than the design strength $S$. In WSD and LSD approaches, this means verifying the condition of Eq. (1), where $F$ is a safety factor, whose value is given based on past experience (usually greater than one) to reduce $S$ to 
account for the uncertainties that are not considered explicitly in these approaches (Prentice 1970).

$$
L \leq \frac{S}{F}
$$

Despite WSD is commonly used in the oil and gas industry because of its simplicity, it has many limitations, such as the lack of risk quantification and the lack of consideration of any event-consequence relationship in the use of $F$ (i.e., all failure modes, as well as their consequences, are treated in the same manner), especially when the design is done for HPHT or deep-water oil wells where past experience is very limited. Another limit of WSD is the use of API burst strength (see Table 1) that leads to overconservative design and excessive cost (Adams et al. 1998).

LSD limits the excessive over-conservative design of WSD, using the casing rupture burst strength (see Table 1) even though also in this approach safety factors are used to assess the structural safety, because uncertainties in load and strength values are not considered.

The RBD approach explicitly treats the uncertainties by considering the probability distributions that affect the uncertain parameters $\bar{x}$ of $S$ and $\bar{y}$ of $L$. When the parameters probability distributions affecting the load cannot be assumed, due to lack of knowledge, it is prudent to resort to the RBPD approach that is a particular type of RBD (Adams et al. 1998), where $L$ is deterministic (the same as in WSD and LSD), whereas $S$ is stochastic with its own distribution (see Table 1). In RBPD and RBD, a safety function is, therefore, used for the structural safety verification of step 4), defined as:

$M(\bar{x}, \bar{y}, t, \tau, d)=S(\bar{x}, t, d)-L(\bar{y}, \tau, d)$

where $M(\bar{x}, \bar{y}, t, \tau, d)$ is the (probabilistic) safety margin (Di Maio et al. 2016) at time $t$ and depth $d$, equal to the difference between the safety variable (in this case $S(\bar{x}, t, d)$ ) limited from below by a lower threshold limit (in this case $L(\bar{y}, \tau, d)$, where $\tau$ is the time of the kick onset). Therefore, the probability of failure $P_{f}(t, \tau, d)$ of the casing at depth $d$ and its reliability can be defined, respectively:

$P_{f}(t, \tau, d)=P(M(\bar{x}, \bar{y}, t, \tau, d)<0)$

$R(t, \tau, d)=1-P_{f}(t, \tau, d)$

Table 1 Design approaches

\begin{tabular}{|c|c|c|}
\hline Method & $L$ (kick load) & $S$ (burst strength) \\
\hline WSD & \multirow{3}{*}{$\begin{array}{l}\text { Deterministic value } \\
\qquad L=P^{i}-P^{o} \\
\qquad \text { (Blade Energy Partners 2005) } \\
\text { where: } \\
P^{i} \text { casing internal pressure at depth } d \\
P^{o} \text { casing external pressure at depth } d\end{array}$} & $\begin{array}{l}\text { Deterministic value } \\
\qquad S=0.875 \cdot\left(\frac{2 \sigma_{y} w}{O D}\right) \\
\text { where: } \\
\sigma_{y} \text { yield strength } \\
w \text { casing wall thickness } \\
O D \text { casing outside diameter } 5 \mathrm{C} 31985)\end{array}$ \\
\hline LSD & & $\begin{array}{l}\text { Deterministic value } \\
\qquad S=0.875 \frac{2 \cdot \sigma_{u} \cdot w}{(O D-w)} \\
\text { where: } \quad \text { Wu et al. 2005) } \\
\sigma_{u} \text { ultimate tensile strength } \\
w \text { casing nominal wall thickness }\end{array}$ \\
\hline RBPD & & \multirow{2}{*}{$\begin{array}{l}\text { Stochastic value } \\
\qquad S(\bar{x}, t, d)=\frac{2 \cdot \sigma_{u} \cdot w}{(O D-w)} \cdot\left(\frac{w_{r}(t, d)}{w}\right) \\
\text { (Wu et al. 2005) } \\
\bar{x}=\left[\sigma_{u}, w, O D\right] \\
w_{r}(t, d) \text { casing remaining wall thickness }\end{array}$} \\
\hline RBD & $\begin{array}{l}\text { Stochastic value } \\
\text { Field load data are used to estimate the prob- } \\
\text { ability distributions of the stochastic varia- } \\
\text { bles that affect the load (e.g., kick volume, } \\
\text { kick intensity, etc.) (Adams et al. 1998) }\end{array}$ & \\
\hline
\end{tabular}


The primary objective of this work is to improve the RBPD approach for a realistic risk-based design of a casing string by accounting not only for the uncertainties affecting $S$ but also for the unlike occurrence of a kick load phenomenon, by calculating the evolution of safety margins at different well depths, within a Risk-Informed Safety Margin Characterization (RISMC) design framework (Di Maio et al. 2016). In practice, we use the probabilistic safety margins to determine the probability of failure of a section of a given casing string design during the drilling phase, or, vice versa, the probabilistic safety margins might be used to retroact the design for satisfying requested safety levels.

In what follows, Section 2 presents the method for a probabilistic design based on Order Statistic (OS) (Nutt et al. 2004) (Martorell et al. 2006) (Martorell et al. 2009). Section 3 introduces the casing string layout and the risk assessment method. Section 4 presents the results of the application of the approach and, then, in Section 5 conclusion are drawn.

\section{THE METHOD}

The estimation of the probability density function of $M(\bar{x}, \bar{y}, t, \tau, d)$ of Eq. (2) can be computationally very expensive, especially when the degradation mechanisms are to be modelled. In order to reduce the computational burden, we propose to compute only some percentiles of $M(\bar{x}, \bar{y}, t, \tau, d)$, with a limited and controlled number of simulations (Zio et al. 2010) (Zio et al. 2008) (Di Maio et al. 2016). In this case, the confidence in the percentiles estimates becomes crucial for decision making and must, thus, be quantified. In practice, a small number $N$ of realizations $S_{i}\left(\bar{x}_{i}, t, d\right), i=1,2, \ldots, N$, is obtained by drawing $N$ times by Monte Carlo sampling methods (Zio 2013) the values of the parameters in $\bar{x}$ from their respective probability distributions and, then, by running $N$ times the code that simulates the strength evolution in time at depth $d$. These $N$ realizations $S_{i}$ can be used to estimate given percentiles of the distributions of $M(\bar{x}, \bar{y}, t, \tau, d)$ when the onset time $\tau$ of a kick load is also sampled from its own distribution.

To obtain the desired confidence in the safety margin percentile, the number $N$ has to be defined based on Order Statistics (OS) methodology (Nutt et al. 2004), which applies independently from the type of probability distribution. The OS allows $N$ to be kept low because only statistical intervals are estimated and not the full probability distribution.
Practically, the estimation of the percentiles of $M(\bar{x}, \bar{y}, t, \tau, d)$ proceeds as follows: at each time $t$, we collect $N$ values $M_{i}\left(\bar{x}_{i}, \bar{y}, t, \tau, d\right)$ that belong to $M_{\text {sort }}=\left\{M_{1}\left(\bar{x}_{1}, \bar{y}, t, \tau, d\right), M_{2}\left(\bar{x}_{2}, \bar{y}, t, \tau, d\right), \ldots, M_{N}\left(\bar{x}_{N}, \bar{y}, t, \tau, d\right)\right\}$, that is the ascending ordered set of values of $M_{i}\left(\bar{x}_{i}, \bar{y}, t, \tau, d\right)$ when the code has been run $N$ times for $N$ different input vectors $X=\left\{\bar{x}_{1}, \bar{x}_{2}, \ldots, \bar{x}_{N}\right\}$ and one realization $\tau$ of the kick load onset. It is worth mentioning that if the code were run a very large number of times $(N \rightarrow+\infty)$, it could be possible to give a sufficiently accurate estimate of the full distribution of $M(\bar{x}, \bar{y}, t, \tau, d)$ and draw probabilistic conclusions on where it lies with respect to the threshold value $M=0$, that limits the integrity of the casing. Given the computational costs associated with the estimation of the evolution of the full distribution (drawn in continuous line in Figure 1), one is forced to focus on verifying that with some level of confidence $\beta$, a certain percentage $\gamma$ of the calculated values of $M(\bar{x}, \bar{y}, t, \tau, d)$ falls within the safety envelope $M>0$ (i.e., the probabilistic safety margin of $M(\bar{x}, \bar{y}, t, \tau, d)$ is limited from below). The approach proposed aims at showing that if the $m$-th member $M_{m}\left(\bar{x}_{m}, \bar{y}, t, \tau, d\right)$ of the $N$ sorted realizations $M_{\text {sort }}$ is taken as the estimated $\gamma$ -th percentile $\hat{M}_{\gamma}(\bar{x}, \bar{y}, t, \tau, d)$, its probability of being less than the unknown true $\gamma$-th percentile $M_{\gamma}(\bar{x}, \bar{y}, t, \tau, d)$ is equal to $\beta$, as shown in Fig. 1 .

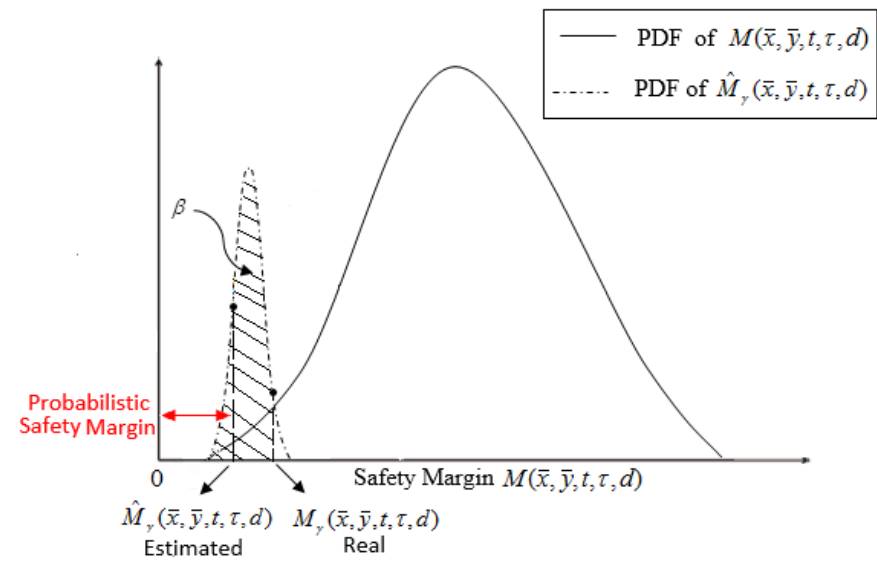

Figure 1 Representation of the (unknown) safety margin probability distribution and its real and estimated $\gamma$-th percentile probability distribution, with confidence $\beta$

In other words, being $\gamma$ and $\beta$ defined as $\gamma=P\left(M(\bar{x}, \bar{y}, t, \tau, d)<M_{\gamma}(\bar{x}, \bar{y}, t, \tau, d)\right) \quad$ and $\beta=P\left(M_{\gamma}(\bar{x}, \bar{y}, t, \tau, d)>\hat{M}_{\gamma}(\bar{x}, \bar{y}, t, \tau, d)\right)$, one has a level of confidence $\beta$ that $M_{\gamma}(\bar{x}, \bar{y}, t, \tau, d)$ is greater 
than the estimated percentile $\hat{M}_{\gamma}\left(\bar{x}_{m}, \bar{y}, t, \tau, d\right)$ : if $\hat{M}_{\gamma}\left(\bar{x}, \bar{y}, t_{k}, \tau, d\right)>0$, then $M_{\gamma}(\bar{x}, \bar{y}, t, \tau, d)>0$, too, as it is show in Fig. 1.

Once $\gamma$ and $\beta$ are fixed, the OS method for calculating the $(\beta \mid \gamma)$-percentile estimate follows the lines of (Zio et al. 2010) and consists in (Nutt et al. 2004):

(i) Determining the sample size $N$ by fixing a positive integer $m$. The probability that at least $m$ observations of a random sample of size $N$ are lower than the $\gamma$-percentile of the distribution generating the sample is

$\beta=\sum_{k=0}^{N-m}\left(\frac{N}{k}\right) \cdot \gamma^{N-k} \cdot(1-\gamma)^{k}$

To find the sample size $N$, Eq. (12) has to be solved in terms of $N$.

(ii) Sorting the observations in the sample by increasing value, the element in the $m-t h$ place being the statistic of order $m$.

(iii) Estimating the $\gamma$-percentile by setting $\hat{M}_{\gamma}(\bar{x}, \bar{y}, t, \tau, d)$ equal to the statistic of order $m$, i.e. the $m$-th smallest observation in the sample;

then

$\beta=P\left(M_{\gamma}(\bar{x}, \bar{y}, t, \tau, d)>\hat{M}_{\gamma}(\bar{x}, \bar{y}, t, \tau, d)\right)$.

Note that higher values of $m$ in step (i) imply higher values of the sample size $N$ but generate less conservative estimates of the $\gamma$-th percentile; in any case, the sample size $N$, i.e. the number of probabilistic safety margin evolution code runs, can be kept low because only intervals related to the $\gamma$-th percentile are estimated and not the full probability distribution generating the data.

In Fig. 2, the flowchart of the procedure to evaluate the probability of casing failure $P_{f}(t, \tau, d)$ at depth $d$ at time $t$, given the kick onset time $\tau$ is sketched.

\section{CASE STUDY}

The objective is to evaluate the probability of failure of a casing string that degrades due to wear and a kick shock. The string under test is the 9-5/8 [in], $\mathrm{L}$ $80,47[\mathrm{lb} / \mathrm{ft}]$ casing string shown in Figure 3, together with its apparent dogleg severity $D L$, that will become useful in what follows. We will consider four casing sections at depths: $d_{1}=1000[\mathrm{ft}$, $d_{2}=2000[\mathrm{ft}], d_{3}=7000[\mathrm{ft}], d_{4}=10000[\mathrm{ft}]$.
The strength of the casing is realistically assumed to reduce due to casing wear (Schoenmakers 1987) (Bradley et al. 1975) (White et al. 1987). This occurs when the drill string tension forces the rotating tool joint against the inner wall of the casing on the concave side of a curve portion of a well path (Hall et al. 1994). The rotating tool joint wears away a crescent volume of the casing, as shown in Figure 4 (Hall et al. 1994). This degradation mechanism results in the unpleasant reduction of the casing remaining wall thickness $w_{r}(t, d)$ and, thus, in the reduction of casing strength $S(\bar{x}, t, d)$.

Several models have been proposed to estimate casing wear, like the wear-efficiency model (White et al. 1987) (Hall et al. 1994), and the nonlinear casing wear model (Sun et al. 2012). In this application, we utilize this latter model because it has been shown to better describe the phenomenon better than the former one (Sun et al. 2012). Accordingly to the nonlinear wear model, the volume $V$ of casing removed (per foot of well depth) $\left[\mathrm{in}^{3} / \mathrm{ft}\right]$ by the tool joint is (Deli et al. 2010):

$V(t, d)=\frac{f_{w}}{\chi(t, d)} \cdot \phi \cdot S D$

where $f_{W}$ is a wear condition coefficient [in/psi], $\chi(t, d)$ is half of the contact width [in], $\phi$ is the lateral load (per foot of well depth) [lbf/ft] equal (Hall et al. 1994):

$\phi=2 \cdot T \cdot \delta_{D L} \cdot \sin \left(\frac{D L}{2}\right)$

where $T$ is the string tension $[\mathrm{lbf} / \mathrm{ft}], D L$ is the apparent dogleg severity [radians/ft] that is computed from well directional survey data, that are taken before or just after setting intermediate casing, $\delta_{D L}$ is a factor that accounts for underestimation of $D L, S D$ is the sliding distance that the tool joint runs against the casing inner wall, that is given by:

$S D=\pi \cdot D_{t j} \cdot 60 \cdot N_{R P M} \cdot t \cdot \frac{L_{t j}}{L_{d p}}$

where $D_{t j}$ is the outside diameter of a tool joint [in], $N_{R P M}$ is the rotary speed of the tool joint [RPM], $t$ is the drilling time [hours], $L_{t j}$ is the length of a tool joint and $L_{d p}$ is the length of one joint of drill pipe. This makes $V$ increasing during drilling time $t$ (see Eq. (5) above), and depending on $d$ since $T$ and $D L$ depend on $d$ (see Eq. (6) above). Thus, at any $t$ and $d$, we can quantify the depth of wear groove $h(t, d)$ using the geometric relationships for a crescentshape wear groove and $w_{r}(t, d)$ (Kumar et al. 2013). 


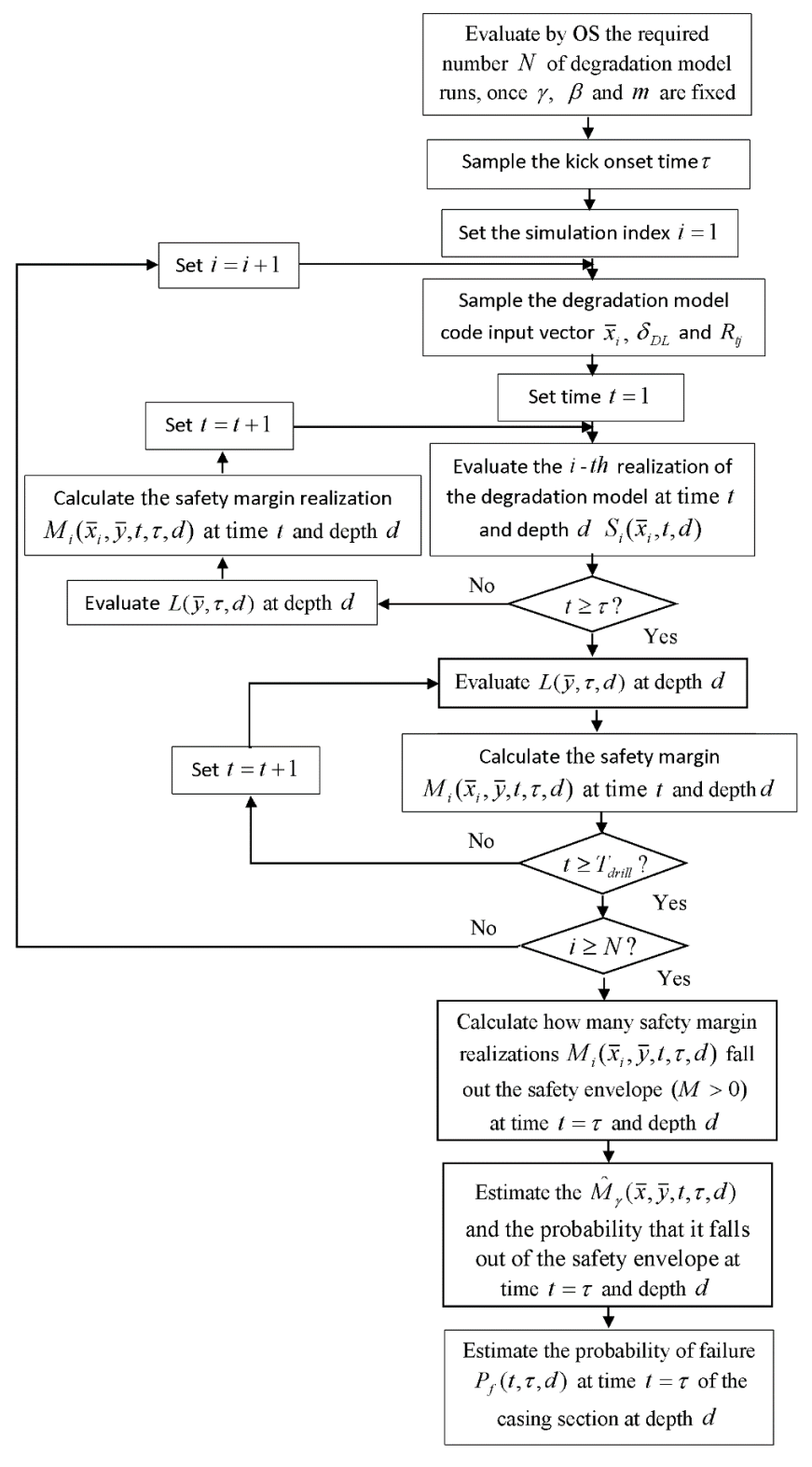

Figure 2 Flowchart of the OS-based RBPD approach

The quantification of $w_{r}(t, d)$ is challenged by the uncertainty that affects some of the parameters involved in the wear model, whose distributions are listed in Table 2. In particular, the largest uncertainty affects $D L$ and this challenges the prediction of the casing wear degradation progression. Indeed, $D L$ is computed from usually inaccurate well survey data influenced by the survey accuracy and survey station spacing, resulting in an underestimation of the actual dogleg severity. To take into account this underestimation, it is recommended to use a multiplication factor $\delta_{D L}$ that increases $\phi$ (see Eq. (5) above (Hall et al. 1994)). As the RBPD approach entails, we consider the variables uncertainties affecting $S$, listed in Table 2, as well as its reduction due to the casing wear, and calculate at each time $t$ the evolution of the safety margin $M(\bar{x}, \bar{y}, t, \tau, d)$.
Regarding the load $L$, we assume a kick load to occur at a random time $\tau$ within the drilling time $T_{\text {drill }}$ . The blowout is an uncontrolled flow of hydrocarbons (e.g., gas and condensate) or even saltwater from a well to the surrounding environment (Khakzad et al. 2013). A kick can result in a blowout if it is not detected in a timely manner and properly prevented by design.
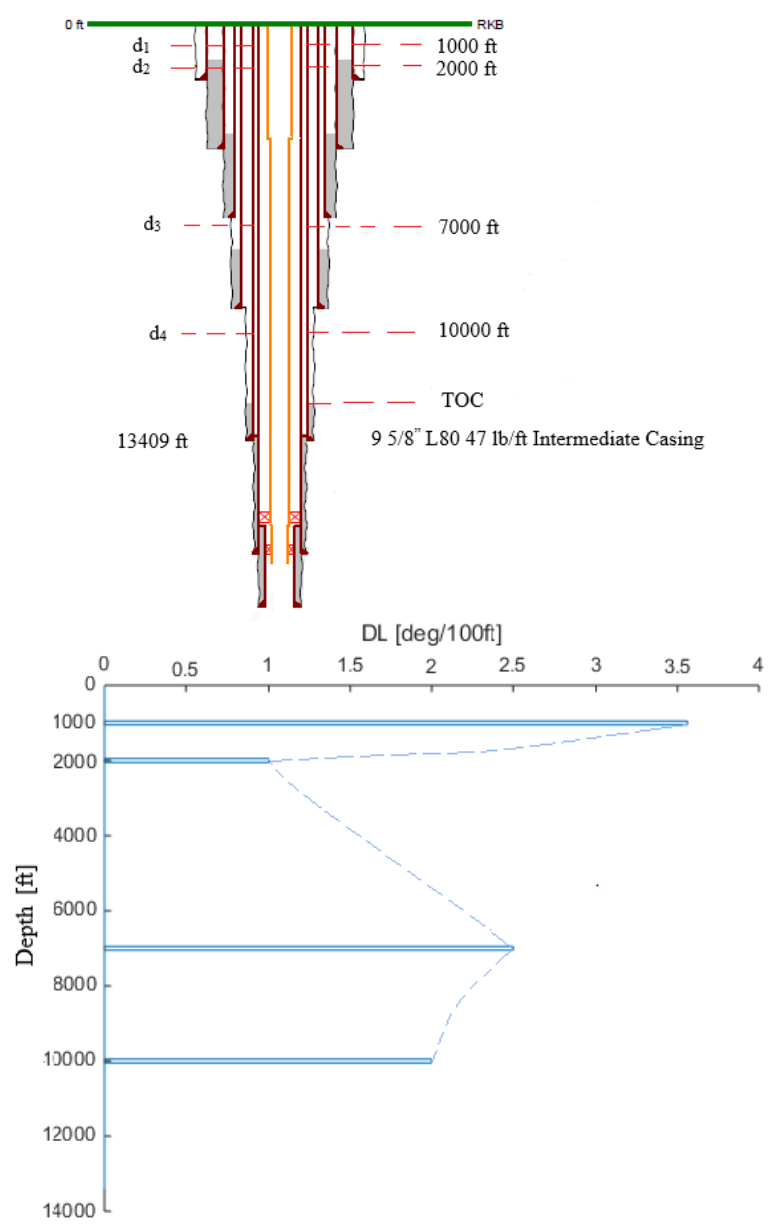

Figure 3 Wellbore schematic with its dogleg severity (adapted from (Blade Energy Partners 2005))

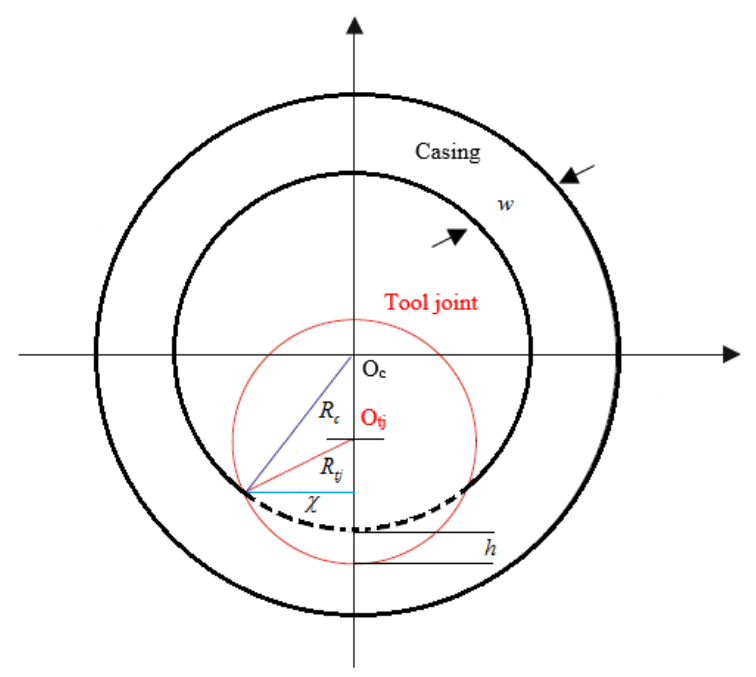

Figure 4 Worn casing geometry (adapted from (Deli et al. 2010)) 
Table 2 List of the stochastic variables considered in the wear model and their uncertainty distributions

\begin{tabular}{|c|c|c|}
\cline { 2 - 3 } \multicolumn{1}{c|}{} & Parameter & Distribution \\
\hline \multirow{4}{*}{$S$} & $O D$ & $\mathrm{~N}(9.635 ; 0.003)$ \\
\cline { 2 - 3 } & $w$ & $\mathrm{~N}(0.479 ; 0.003)$ \\
\cline { 2 - 3 } & $\sigma_{u}$ & $\mathrm{~N}(95000 ; 2800)$ \\
\hline $\begin{array}{c}\text { Casing } \\
\text { Wear Model }\end{array}$ & $\delta_{D L}$ & $\mathrm{U}(1 ; 4)$ \\
\cline { 2 - 3 } & $R_{t j}$ & $\mathrm{~N}(3.25 ; 0.0015)$ \\
\hline
\end{tabular}

\section{$4 \quad$ RESULTS}

The proposed OS-based RBPD approach of Section 2 has been applied to evaluate the probability of casing failure $P_{f}(t, \tau, d)$ considering the kick as an initiating event, the successful kick detection and the success of at least one of the barriers (rams or preventer), while the thickness of the casing is reduced due to wear. Moreover, we have compared the results obtained using Eq. (3) with those provided by WSD and LSD approaches by assessing the casing failure as in Eq. (1). The results of the application of the WSD, LSD and RBPD approaches are presented. For WSD and LSD approaches, we check whether the condition of Eq. (1) is verified at the four casing section depths considered. Instead, according to RBPD method we verify if $M(\bar{x}, \bar{y}, t, \tau, d)$ falls in the safety envelope ( $M>0$, see Section 2). Table 3 resumes the load and strength values used for the calculation of the safety margins under the assumptions of the three methods.

Table 4 shows the safety margin results under the different assumptions of WSD and LSD at the four different well depths, and for a generic realization of the RBPD with one random realization of the kick time $\tau$, sampled from a uniform distribution in the range between the time in which the drilling starts $t=0$ and the time in which it finishes $t=T_{d r i l l}$. At first, we point out that the only approach that considers the time dependency of the strength and the time of occurrence of the kick load is the RBPD approach. The WSD and LSD approaches, instead, provide only a constant estimation of the safety margins. Also, we can notice that sections $d_{1}$ and $d_{2}$ would fail $(M<0)$ according to the WSD approach; conversely, according to the LSD approach, all four casing sections $d_{1}, d_{2}, d_{3}$ and $d_{4}$ are safe. The difference in the results of WSD approach and LSD approach, is due to a strong limit of WSD, that is the use of API burst strength (API Bulletin 5C3 1985) that leads to over-conservative design and excessive cost (Adams et al. 1998); LSD, instead, limits the excessive over-conservative design of WSD, using the casing rupture burst strength of (Wu et al. 2005). Finally, according to the RBPD approach, the margin $M(\bar{x}, \bar{y}, t, \tau, d)$ falls in the safety envelope ( $M(\bar{x}, \bar{y}, t, \tau, d)>0)$ for all four casing sections, and, thus, according to the RBPD methodology, all the four casing sections are safe. These insights are also resumed in Table 4.

Table 3 List of the load and strength values for safety margin calculation

\begin{tabular}{|c|c|c|c|c|c|c|}
\hline & $S(\bar{x}, 0, d)$ & $\begin{array}{c}L \\
\text { at } d_{1}\end{array}$ & $\begin{array}{c}L \\
\text { at } d_{2}\end{array}$ & $\begin{array}{c}L \\
\text { at } d_{3}\end{array}$ & $\begin{array}{c}L \\
\text { at } d_{4}\end{array}$ & $F$ \\
\hline WSD & $\begin{array}{l}6865 \\
{[p s i]}\end{array}$ & \multirow{3}{*}{$\begin{array}{c}7580 \\
{[p s i]}\end{array}$} & \multirow{3}{*}{$\begin{array}{l}7160 \\
{[p s i]}\end{array}$} & \multirow{3}{*}{$\begin{array}{l}4744 \\
{[p s i]}\end{array}$} & \multirow{3}{*}{$\begin{array}{l}3349 \\
{[p s i]}\end{array}$} & \multirow{2}{*}{1.1} \\
\hline LSD & $\begin{array}{l}8573 \\
{[p s i]}\end{array}$ & & & & & \\
\hline RBPD & $\begin{array}{c}\text { Depends on } \\
\text { the sampled } \\
\text { values of } \\
\bar{x} \\
\end{array}$ & & & & & \\
\hline
\end{tabular}

Table 4 Casing design results of the simulations of Fig. 8

\begin{tabular}{|c|c|c|c|}
\hline $\begin{array}{c}\text { Casing } \\
\text { Section }\end{array}$ & WSD & LSD & RBPD \\
\hline$d_{1}$ & $P_{f}\left(t, d_{1}\right)=1$ & Safe & Safe \\
\hline$d_{2}$ & $P_{f}\left(t, d_{1}\right)=1$ & Safe & Safe \\
\hline$d_{3}$ & Safe & Safe & Safe \\
\hline$d_{4}$ & Safe & Safe & Safe \\
\hline
\end{tabular}

In what follows, we focus our attention on the section at $d_{1}$ being the most critical casing section since it is subjected to the highest load and the most severe wear.

By sampling $N$ times the input vector $\bar{x}$, for a given kick time $\tau$, we aim at evaluating a $\gamma$-th percentile with a confidence $\beta$ of the distribution of $M\left(\bar{x}, \bar{y}, t, \tau, d_{1}\right)$. We evaluate the number $N$ for estimating the 5 -th percentile of the distribution of $M\left(\bar{x}, \bar{y}, t, \tau, d_{1}\right)$, with $\beta=0.95$, which is $N=59$ (Nutt et al. 2004). Fig. 5 shows the 59 evolutions of the safety margins when the kick load time is $\tau=58$ [hours]. One might misleadingly conclude that, since 
the probability of failure $P_{f}\left(t, \tau=58, d_{1}\right)=0$, the design of the casing string is safe at any time of occurrence of the kick load. Indeed, the wear evolution strongly impacts the safety margin evolution in time, as it can be seen in Fig. 6, where $N=148$ and $\tau$ is sampled one hundred times.

It can be seen, that the probability of casing failure is not equal to zero, but rather, a given percentile of the distribution of $M\left(\bar{x}, \bar{y}, t, \tau=123, d_{1}\right)$ becomes negative at $t=123$ [hours]. To calculate the percentile at which, at any time, $M\left(\bar{x}, \bar{y}, t, \tau, d_{1}\right)$ falls out the safety envelope, we could run the model $N=+\infty$ times. This is obviously infeasible and to avoid this, we provide by the OS theory the estimate of the percentile $\gamma$ for which $M\left(\bar{x}, \bar{y}, t, \tau=123, d_{1}\right)<0 \quad$ with a confidence $\beta=0.95$. This, for example, is equal to $\gamma=0.032$ for $t=123$ [hours] (because $m=2$ simulations provide negative safety margin results, shown with dots in the zoom of Fig. 6). Therefore, since the 3.2-th percentile of the safety margin probability distribution evolution $M\left(\bar{x}, \bar{y}, t, \tau=123, d_{1}\right)$ becomes lower than 0 at time $t=123$ [hours] for the casing section $d_{1}=1000$ [ft], we can estimate with a confidence $\beta=0.95$ that the probability of casing failure at depth $d_{1}$ is $P_{f}\left(t=123, \tau=123, d_{1}\right) \leq 0.032$, in this scenario when a kick load occurs, the kick detection system correctly detects the anomaly and at least one of the barriers succeed in controlling the blowout

Similarly, we can estimate $P_{f}\left(t, \tau, d_{1}\right)$ at each time $t \geq 123$ [hours] and use this time-dependent probability to calculate the blowout probability of the casing section at depth $d_{1}$ (plotted in Fig. 7). It is worth mentioning that the computation of the overall blowout probability requires modelling the casing string as a series of casing sections that may independently fail. In other words, assuming to subdivide the casing string into $\Omega$ sections, $\alpha=1,2,3, \ldots . \Omega$, each one characterized by a given $P_{f}\left(t, \tau, d_{\alpha}\right)$, the overall casing string failure probability is equal to:

$$
P_{f, \text { vverall }}(t, \tau)=1-\prod_{\alpha=1}^{\Omega}\left(1-P_{f}\left(t, \tau, d_{\alpha}\right)\right)
$$

\section{CONCLUSION}

Deep-water wells for oil and gas extraction make structural components, such as casing and tubing, work in extremely harsh environmental conditions that accelerate component degradation and increase failure probability. In this work, we propose to properly design casing strings under these operative circumstances with a Resistance-Based Probabilistic
Design (RBPD) approach that is informed with the quantification of the probabilistic safety margin that the design load does not exceed the casing strength. Wear is taken and modelled as the main degradation mechanism during drilling and the accidental event of a kick load is considered to affect the performance of the casing string.

On a real case study, we have shown that the WSD approach is more conservative than the LSD approach, resulting in different design conclusions. However, this latter approach still lacks of a proper treatment of the uncertainties affecting the reliability of the casing sections. To inform this with the proper confidence, we have evaluated the probabilistic safety margins of the casing sections during drilling with the OS-based RBPD approach here proposed. Results can be useful to control the process during drilling or to retroact on the design in order to achieve the acceptable level of casing probability of failure.

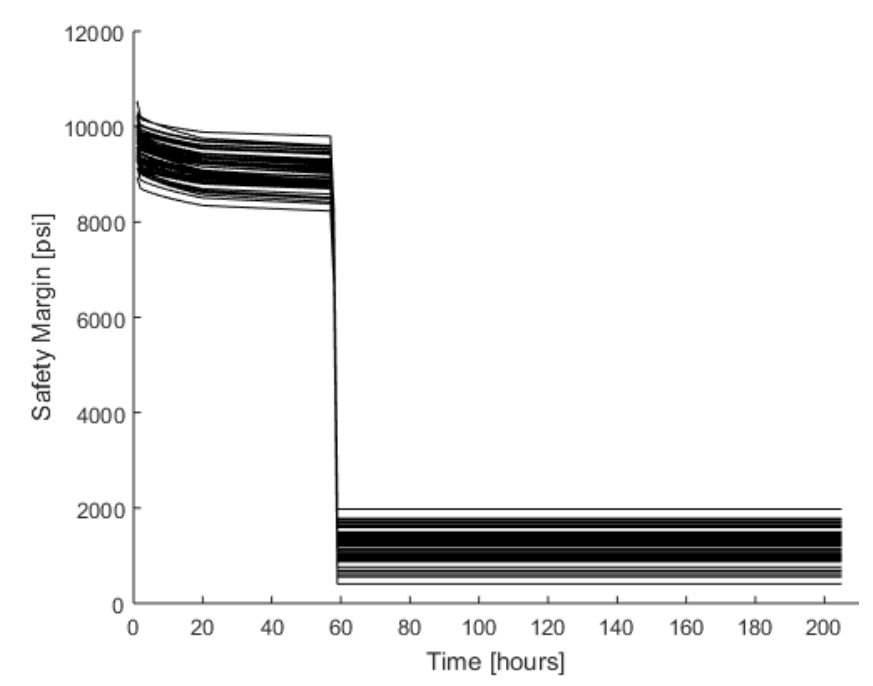

Figure 5 Safety margin of first casing section considering 59 simulations and one kick time

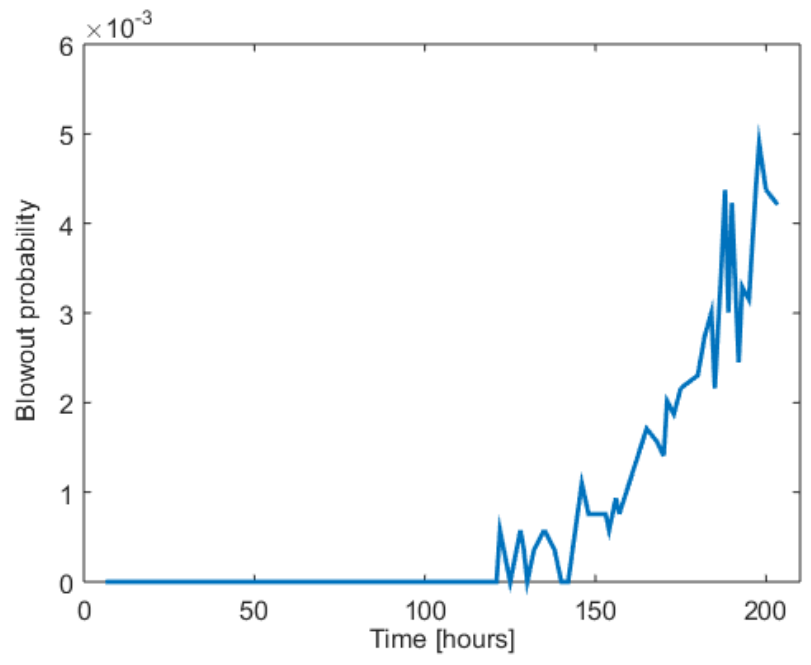

Figure 7 Blowout probability of the casing at depth $d_{1}$ 


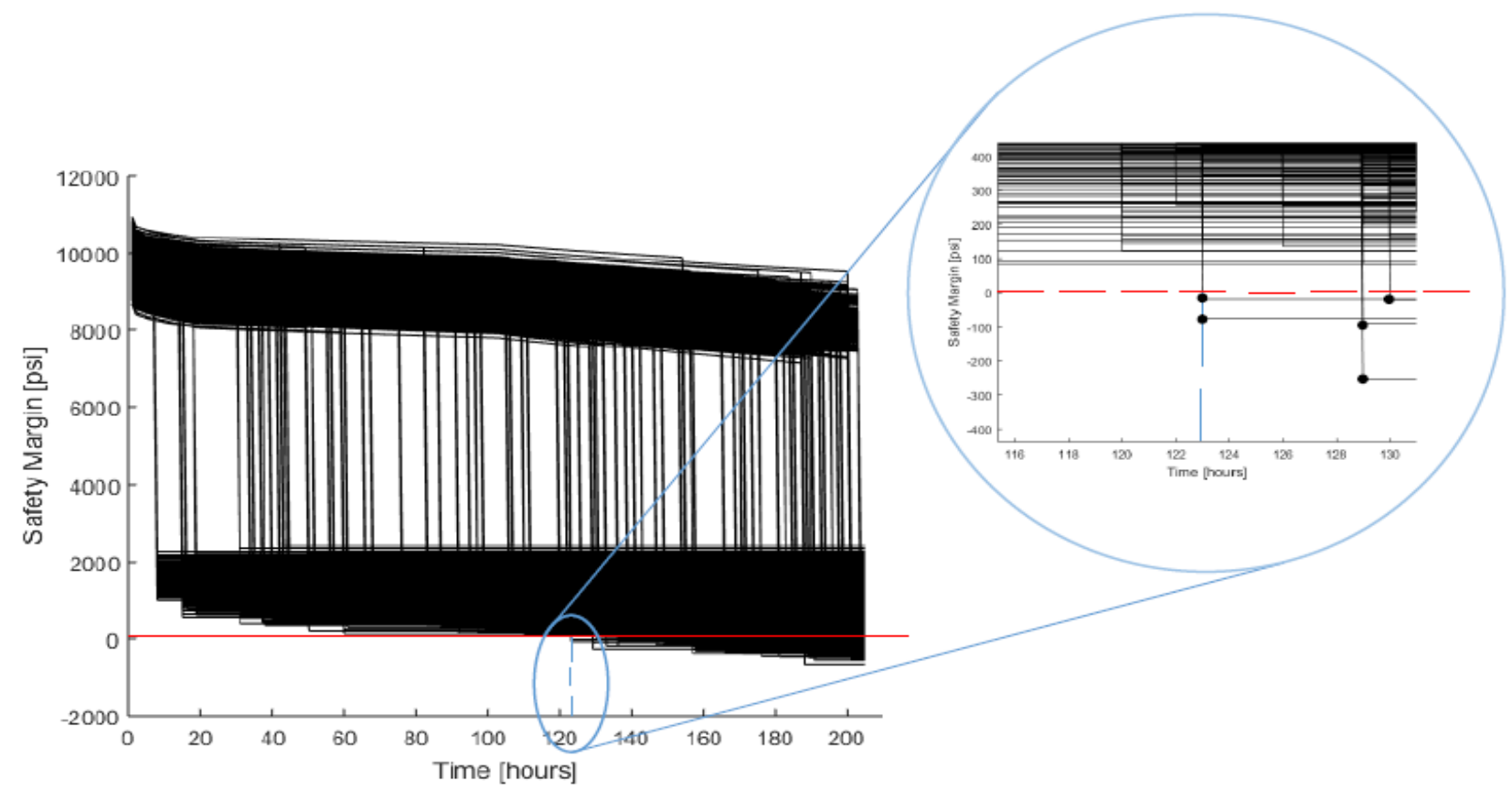

Figure 6 Safety margin of the first casing section at depth $d_{1}$ considering 148 simulations and 100 kick time

\section{REFERENCES}

Abimbola, M., Khan, F. \& Khakzad, N. 2014. Dynamic safety risk analysis of offshore drilling. J. Loss Prev. Process Ind., vol. 30, pp. 74-85.

Adams, A.J. \& Glover, S.B. 1998. An Investigation Into the Application of QRA in Casing Design. Presented at SPE Applied Technology Workshop on Risk Based Design of Well Casing and Tubing, The Woodlands, Texas, 7-8 May

API Bulletin 5C3 1985. Bulletin on Formulas and Calculation for Casing, Tubing, Drill Pipe, and Line Pipe Properties, American Petroleum Institute, 1220 L Street, Washington, D.C.

Baraldi, P., Di Maio, F., Sauco, S., Zio, E., Droguett, E. \& Magno, C. 2012a. Ensemble of Neural Networks for Predicting Scale Deposition in Oil Well Plant Equipments. proceedings of PSAM11 \& ESREL 2012 conference, ISBN 978162-276-4365, Helsinki, Finland, 25-29 June

Baraldi, P., Di Maio, F., Sauco, S., Zio, E., Droguett, E. \& Magno, C. 2012b. Sensitivity analysis of scale deposition on equipment of oil wells plants. Chem. Eng. Trans., vol. 26, pp. 327-332.

Blade Energy Partners 2005. Principles and Practice of Casing Design.

Bradley, W.B. \& Fontenot, J.E. 1975. The Prediction and Control of Casing Wear. SPE Journal of Petroleum Technology, vol 27, pp. 233-245.

Deli, G., Lianzhong, S. \& Jihong, L. 2010. Prediction of casing wear in extended-reach drilling. Petroleum Science, vol 7 (4), pp. 494-501

Di Maio, F., Rai, A. \& Zio, E. 2016. A dynamic probabilistic safety margin characterization approach in support of Integrated Deterministic and Probabilistic Safety Analysis. Reliab. Eng. Syst. Saf., vol. 145, pp. 9-18.

Hall, R.W., Gartil, W., Deskims, G. \& Vozniak, J. 1994. Recent Advances in Casing Wear Technology. Presented at IADC/SPE Driliing Conference, 15-16 February, Dallas, Texas, Paper No. SPE 27532

Khakzad, N., Khan, F., \& Amyotte, P. 2013. Quantitative risk analysis of offshore drilling operations: A Bayesian approach. Saf. Sci., vol. 57, pp. 108-117.

Kumar, A., Nwachukwu, J. \& Samuel, R. 2013. Analytical Model to Estimate the Downhole Casing Wear Using the Total Wellbore Energy. J. Energy Resour. Technol, vol. 135 (4), p. 042901
Martorell, S., Nebot, Y., Villanueva, J.F., Carlos, S., Serradell, V., Pelayo, F. \& Mendizàbal, R. 2006. Safety margins estimation method considering uncertainties within the risk- informed decision-making framework. In: Proceedings of the PHYSOR 2006 conference, Vancouver, Canada, 10-14 September.

Martorell, S., Nebot, Y., Villanueva, J.F., Carlos, S., Serradell, V., Pelayo, F. \& Mendizàbal, R. 2009. An approach to integrate thermal-hydraulic and probabilistic analyses in addressing safety margins estimation accounting for uncertainties. Safety, reliability and risk analysis:theory, methods and applications.In: Proccedings of the Joint ESREL and SRA-Europe conference, vol. 4, pp. 2827-2835.

Mitchell. S., \& Xiang, Y. 2012. Improving Casing Wear Prediction and Mitigation Using a Statistically Based Model. IADC/SPE 151448 Drilling Conference and Exhibition vol. 100 , pp. 1-15.

Nutt, W.T. \& Wallis, G.B., 2004. Evaluation of nuclear safety from the outputs of computer codes in the presence of uncertainties. Reliab. Eng. Syst. Saf., vol. 83, no. 1, pp. 57-77.

Prentice, C. 1970“"Maximum Load' Casing Design," J. Pet. Technol., vol. 22, pp. 805-811.

Schoenmakers, J.M. 1987. Casing Wear During Drilling : Simulation, Prediction, and Control. SPE Drilling Engineering, vol. 2, pp. 375-381.

Sun, L., Gao, D. \& Zhu, K. 2012. Models \& tests of casing wear in drilling for oil \& gas. J. Nat. Gas Sci. Eng., vol. 4, pp. 44-47.

White, J.P. \& Dawson, R. 1987. Casing Wear: Laboratory Measurements and Field Predictions. SPE Drilling Engineering, vol 2 (1)., pp. 56-62.

Wu, J. \& Zhang, M.G. 2005. Casing Burst Strength After Casing Wear. Presented at SPE production operations symposium, Oklahoma City, Oklahoma, 16-19 April.

Zio, E. \& Di Maio, F. 2008. Bootstrap and Order Statistics for Quantifying Thermal-Hydraulic Code Uncertainties in the Estimation of Safety Margins. Sci. Technol. Nucl. Install., vol. 2008, pp. 1-9.

Zio, E., Di Maio, F., \& Tong, J. 2010. Safety margins confidence estimation for a passive residual heat removal system. Reliab. Eng. Syst. Saf., vol. 95 (8), pp. 828-836.

Zio, E. 2013. The Monte Carlo Simulation Method for System Reliability and Risk Analysis. ISBN 978-1-4471-4588-2, London: Springer-Verlag. 